\title{
Norms of Best Smoothness in Orlicz Spaces
}

\author{
R.P. MALEeV
}

Equivalent norms of best smoothness are constructed for large classes of Orlicz sequence and function spaces.

Key words: Orlicz spaces, Frechet differentiability

AMS subject classification: $46 \mathrm{~B} 20,46 \mathrm{E} 30$

\section{Introduction}

It is well known $[1,12]$ that the usual norm in the spaces $l_{p}$ and $L_{p}(p>1, p$ not even) is $E(p)$ times uniformly differentiable, and the Taylor remainder term is of order $p-E(p)$, where

$$
E(p)= \begin{cases}p-1 & \text { if } p \text { is an integer } \\ {[p]} & \text { otherwise }\end{cases}
$$

Moreover, this order can not be improved by equivalent renorming [1]. For the Orlicz spaces $l_{M}$ and $L_{M}$, the exact order, up to equivalent Orlicz renorming, of the reminder term after differentiation of the norm was found in [9]. This order gives information about the type of $l_{M}$, $L_{M}$ (see, e.g., [6: Section 1.e.16]).

Recently, the best order of Frechet and uniformly Frechet differentiability of the norm (up to equivalent renorming) in Orlicz sequence and function spaces was found in [10]. As usual, in every case an appropriate Orlicz function is constructed so the corresponding Orlicz norm, equivalent to the initial one, is of highest order of differentiability. Our aim is a further investigation of the smoothness of this "good" norm, which is related to a precise estimation of the remainder term after the last derivative. It turns out that in many cases it is also norm of best smoothness. We note that, in a separable Banach space, the existence of an equivalent norm (or more generally bump function) from some smoothness class implies the existence of a partition of unity from the same class (see, e.g., [13: Section 3.1.6]).

Some of the results contained in this paper were announced in a talk given by the author at the 17-th Winter School on Abstract Analysis, Srni, CzechoSlovakia, 1990.

\section{Preliminaries}

We begin with some notations and definitions. In the sequel $X, Y$ denote Banach spaces, $S(X)$ the unit sphere of $X, B(x ; r)$ the ball centered at $x$ of radius $r$, and $N$ the set of all naturals, $R$ of all reals, $\mathbf{R}^{+}$of all positive reals. Everywhere differentiability is understood in Frechet sense.

R.P. Maleev: Acad. Sci., Inst. Math. \& Comp. Centre, 8 G. Bonchev, 1090 Sofia, Bulgaria Res. supp. in part by the Bulg. Ministry of Educ. and Sci., Grant No MM-3/91

ISSN $0232-2064$

(C) 1993 Heldermann Vorlag Borlin 
We denote by $B^{j}(X, Y)$ the space of all continuous symmetric $j$-linear forms

$$
T: \underbrace{X \times X \times \ldots \times X}_{j-\text { times }} \rightarrow Y
$$

equipped with the norm

$$
\|T\|_{1}=\sup \left\{\left\|T\left(x_{1}, \ldots, x_{j}\right)\right\| ; x_{i} \in X,\left\|x_{i}\right\| \leq 1(i=1, \ldots, j)\right\} .
$$

In the next we use the notation $x^{(j)}=(\underbrace{x, \ldots, x}_{j-\text { times }})$ for $x \in X$. An equivalent norm (see, e.g., [13: Section 1.3.8]) is given by

$$
\|T\|=\sup \left\{\left\|T\left(x^{(j)}\right)\right\| ; x \in X,\|x\| \leq 1\right\}
$$

and $\|T\| \leq\|T\|_{1} \leq \kappa\|T\|, \kappa=(2 j)^{j} / j !$. If $Y=\mathbf{R}$, the space of all continuous symmetric $j$-linear functionals on $X$ is denoted $B^{j}(X)$.

Definition 1: A map $f: X \longrightarrow Y$ is said to be $k$-times differentiable at $x \in X$ if there exist $T_{j} \in B^{j}(X, Y)(j=1, \ldots, k)$ such that

$$
f(x+t h)=f(x)+\sum_{j=1}^{k} \frac{t^{j}}{j !} T_{j}\left(h^{(j)}\right)+o_{x}\left(|t|^{k}\right)
$$

uniformly for $h$ in the unit sphere $S(X)$ of $X$, i.e. given $\varepsilon>0$ there is a $\delta>0$ independent of $h \in S(X)$ such that $\left|f(x+t h)-\sum_{j=0}^{k} \frac{i^{j}}{j !} T_{j}\left(h^{(j)}\right)\right|<c(x) \varepsilon|t|^{k}$ provided $|t|<\delta . T_{j}$ is called $j$-th derivative of $f$ at $x$ and is denoted $D^{j} f(x)$ or $f^{(j)}(x)$.

Let $\omega: \mathbf{R}^{+} \longmapsto \mathbf{R}^{+}$be a non-decreasing function with $\lim _{t \rightarrow 0} \omega(t)=0$, and $k$ the greatest integer such that $\omega(t)=o\left(t^{k}\right)$. Set $\omega_{1}(t)=\omega(t) / t^{k}$.

Definition 2: A map $f: X \longmapsto Y$ is called $H^{\omega}$-smooth in $V \subset X$ (see, e.g., [2]) if $f$ is $k$-times continuously differentiable and for every $x \in V$ there exist $\delta, A(x)>0$ such that

$$
\left\|f^{(k)}(y)-f^{(k)}(z)\right\| \leq A(x) \omega_{1}(\|y-z\|),
$$

provided $y, z \in B(x ; \delta) \cap V$.

The norm in the left-hand side of (1) is understood as the norm of the $k$-linear continuous symmetric form $f^{(k)}(y)-f^{(k)}(z)$ from $B^{k}(X, Y)$. The space of all $H^{\omega}$-smooth functions on $V$ is denoted $H^{\omega}(V, Y)$. If the norm in a Banach space is $H^{\omega}$-smooth in $X \backslash\{0\}$, then $X$ is called $H^{\omega}$-smooth. A $H^{\omega}$-smooth map (space) with $\omega(t)=t^{p}$ is called $H^{p}$-smooth.

Let us recall some definitions and facts about Orlicz spaces which will be necessary in what follows. An even convex continuous function $M$, defined and non-decreasing on $[0, \infty)$, is called 
Orlicz function if $M(0)=0, M(\infty)=\infty$. Let $(S, \Sigma, \mu)$ be a measure space. The space of all equivalent classes of $\mu$-measurable functions $x$ on $S$ such that

$$
\int_{S} M(x(s) / \lambda) d \mu(s)=\widetilde{M}(x / \lambda)<\infty
$$

for some positive $\lambda$ with the norm

$$
\|x\|=\inf \{\lambda>0 ; \bar{M}(x / \lambda) \leq 1\}
$$

is a Banach space, which is called the Orlicz space generated by $M$ and denoted by $L_{M}(S, \Sigma, \mu)$. The subspace of $L_{M}(S, \Sigma, \mu)$. which consists of all $x$ such that $\bar{M}(\lambda x)<\infty$ for every $\lambda>0$ is denoted $H_{M}(S, \Sigma, \mu)$.

The most interesting Orlicz spaces considered usually in the literature are the sequence spaces $l_{M}, h_{M}$ and the pairs of function spaces $L_{M}(0,1), H_{M}(0,1)$ and $L_{M}(0, \infty), H_{M}(0, \infty)$ corresponding to the cases: $S$ is a countable union of atoms of equal mass, $S=[0,1]$ or $S=$ $[0, \infty)$, and $\mu$ the usual Lebesgue measure. We note that if the Orlicz function $M$ satisfies the $\Delta_{2}$-condition at 0 (at $\infty$, at 0 and $\infty$ ), i.e. there exists $k>0$ such that

$$
M(2 t) \leq k M(t), t \in[0,1] \quad(t \in[1, \infty), t \in[0, \infty)),
$$

the spaces $l_{M}$ and $h_{M}\left(L_{M}(0,1)\right.$ and $H_{M}(0,1), L_{M}(0, \infty)$ and $\left.H_{M}(0, \infty)\right)$ coincide. Obviously $l_{M}, L_{M}(0,1)$ and $L_{M}(0, \infty)$ essentially depend on the behaviour of the function $M$ near $0, \infty$, and 0 and $\infty$, respectively. It is well known (see, e.g., [5]) that if two Orlicz functions $M$ and $N$ are equivalent $(M \sim N)$ at $0($ at $\infty$, at 0 and $\infty)$, i.e.

$$
c^{-1} M\left(c^{-1} t\right) \leq N(t) \leq c M(c t), t \in[0,1](t \in[1, \infty), t \in[0, \infty))
$$

for some positive constant $c$, then $h_{N}\left(H_{N}(0,1), H_{N}(0, \infty)\right)$ is isomorphic to $h_{M}\left(H_{M}(0,1), H_{M}(0, \infty)\right)$. Using this result equivalent norms in $h_{M}, H_{M}(0,1)$ or $H_{M}(0, \infty)$ are usually constructed through Orlicz functions, equivalent to $M$ at 0 , at $\infty$ or at 0 and $\infty$, respectively.

Now we recall that the Boyd indices for $h_{M}, H_{M}(0,1)$ and $H_{M}(0, \infty)$ can be expressed by the formulas (see, e.g., [6: Section 2.b.5])

$$
\begin{aligned}
\alpha_{M}^{0} & =\sup \left\{p ; \sup \left\{\frac{M(u v)}{u^{p} M(v)} ; u, v \in(0,1]\right\}<\infty\right\}, \\
& :=\sup \left\{p ; \sup \left\{\frac{u^{p} M(v)}{M(u v)} ; u, v \in[1, \infty)\right\}<\infty\right\}, \\
\alpha_{M}^{\infty} & =\sin \left(\alpha_{M}^{0}, \alpha_{M}^{\infty}\right),
\end{aligned}
$$

respectively.

A detailed study of the problem of the isomorphic embeddings of the $h_{M}$ spaces into Orlicz spaces is contained in [7] and [8]. Here we only mention that always $\alpha_{M} \geq 1$ and that $h_{\alpha_{M}^{0}}$ is isomorphic to a subspace of $h_{M}$. Finally we consider a class of smooth Orlicz functions that was introduced in [10].

Definition 3: $A C^{k}, k \in \mathbf{N}$ is the class of all functions $M$ such that: 
i) $\alpha_{M}>k_{\text {; }}$

ii) the $k$-th derivative $M^{(k)}$ of $M^{A}$ is absolutely cuintinuous in every finite interval;

iii) $t^{k+1}\left|M^{(k+1)}(t)\right| \leq c M(c t)$ a.e. in $[0, \infty)$ for some $c>0$.

It is not hard to check that every $M \in A C^{k}$ satisfies for fixed $a \in\left(k, \alpha_{M}\right)$ the inequalities

$$
M(\lambda t) \leq c_{1} \lambda^{a} M(t), \lambda \in[0,1], t \in[0, \infty)
$$

and

$$
t^{i}\left|M^{(i)}(t)\right| \leq c_{1} M\left(c_{1} t\right), t \in[0, \infty)(i=1, \ldots, k),
$$

where $c_{1}$ is a constant depending on $a$ and $M$. Without loss of generality we shall assume in the sequel that $c_{1}=c \geq 1$, i.e. that for a fixed $a \in\left(k, \alpha_{M}\right), M$ satisfies (2), (3) and (4) with the same constant $c \geq 1$.

\section{Properties of the class $A C^{k}$}

In this section we make a further investigation of the class $A C^{k}$ in order to improve some estimates from [10]. We shall often use the following simple inequalities implied by the convexity of $\mathrm{M}$ :

$$
\begin{gathered}
u M(v) \leq M(u v)+M(v), \\
M(\max (|u|,|v|)) \leq(M(2 u)+M(2 v)) / 2
\end{gathered}
$$

for any real $u, v$.

Let $k \in \mathbf{N}$. Put

$$
F_{k}^{M}(u, v)=M(u v)\left(u^{k+1} M(v)\right)^{-1} .
$$

For any $k \in \mathrm{N}$ and interval $I \subset \mathrm{R}^{+}$we associate to $M$ the function $r_{k, I}^{M}$ defined as

$$
r_{k, I}^{M}(t)=t \sup \left\{F_{k}^{M}(u, v) ;(u, v) \in[t, 1] \times I\right\} .
$$

If $I=\mathbf{R}^{+}$, we simply choose $r_{k, I}^{M}=r_{k}^{M}$. We set $R_{k, I}^{M}(t)=t^{k} r_{k, I}^{M}(t)$ and $R_{k}^{M}(t)=t^{k} r_{k}^{M}(t)$. Obviously

$$
\begin{gathered}
r_{k}^{M}(t) \geq t, r_{k}^{M}(t) \geq M(t) / t^{k}, t \in(0,1], \\
M(u v) \leq u^{k+1} M(v) r_{k}^{M}(t) / t, t \in(0,1],(u, v) \in[t, 1] \times R^{+} .
\end{gathered}
$$

The following properties of $R_{k}^{M}$ will be useful.

Lemma 1: Let $M \in A C^{k}$. Then $r_{k}^{M}$, and of course also $R_{k}^{M}$, are non-decreasing in $\left[0, t_{0}\right]$ for some $t_{0} \in(0,1)$.

Proof: According to (3) for a suitable $t_{0} \in(0,1)$

$$
M(u v) \leq u^{k} M(v),(u, v) \in\left[0, t_{0}\right] \times \mathbf{R} .
$$

First we show that $r_{k}^{M}(t) \leq r_{k}^{M}(a)$ for any $t \in\left[a^{2}, a\right]$ and $a \in\left(0, t_{0}\right]$. Indeed, the above inequality implies

$$
\sup \left\{F_{k}^{M}(u, v) ;(u, v) \in[t, a] \times \mathbf{R}^{+}\right\} \leq\left(\frac{a}{t}\right) \sup \left\{F_{k}^{M}(u, v) ;(u, v) \in\left[a, \frac{a^{2}}{t}\right] \times \mathbf{R}^{+}\right\}
$$


with $a^{2} / t \leq 1$. Using this inequality and the representation

$$
r_{k}^{M}(t)=t \max \left(\sup \left\{F_{k}^{M}(u, v) ;(u, v) \in[t, a] \times \mathbf{R}^{+}\right\}, \sup \left\{F_{k}^{M}(u, v) ;(u, v) \in[a, 1] \times \mathbf{R}^{+}\right\}\right)
$$

we immediately obtain $r_{k}^{M}(t) \leq r_{k}^{M}(a)$. Let now $0<t_{1}<t_{2} \leq t_{0}$. Then $t_{2}^{2^{j}} \leq t_{1} \leq t_{2}^{2^{j-1}}$ for some $j \in \mathbf{N}$ and the sequence of inequalities

$$
r_{k}^{M}\left(t_{1}\right) \leq r_{k}^{M}\left(t_{2}^{j-1}\right) \leq r_{k}^{M}\left(t_{2}^{2 j-2}\right) \leq \cdots \leq r_{k}^{M}\left(t_{2}\right)
$$

completes the proof.

We note that $r_{k}^{M}(\lambda t) \leq \lambda r_{k}^{M}(t), \lambda \geq 1$.

Lemma 2: If $M \sim N$ at 0 and $\infty$, then $R_{k}^{M} \sim R_{k}^{N}$ at 0 .

Proof: Without loss of generality we may assume that $M(1)=N(1)=1$. Let $c^{-1} M\left(c^{-1} t\right) \leq N(t) \leq c M(c t)$ for some $c \geq 1$. Then

a) for $c^{-2} \leq u \leq 1, v \in \mathbf{R}^{+}$,

$$
\frac{N(u v)}{u^{k+1} N(v)} \leq c^{2(k+1)} \frac{M\left(u_{1} v_{1}\right)}{u_{1}^{k+1} M\left(v_{1}\right)}, \quad u_{1}=v_{1}=1
$$

b) for $t \leq u \leq c^{-2}, v \in \mathbf{R}^{+}$,

$$
\frac{N(u v)}{u^{k+1} N(v)} \leq \frac{c^{2} M(c u v)}{u^{k+1} M\left(c^{-1} v\right)}=c^{2(k+2)} \frac{M\left(u_{1} v_{1}\right)}{u_{1}^{k+1} M\left(v_{1}\right)}, t \leq c^{2} u=u_{1} \leq 1, v_{1}=c^{-1} v
$$

which implies

$$
\sup \left\{F_{k}^{N}(u, v) ;(u, v) \in[t, 1] \times \mathbf{R}^{+}\right\} \leq c^{2(k+2)} \sup \left\{F_{k}^{M}(u, v) ;(u, v) \in[t, 1] \times \mathbf{R}\right\}
$$

and symmetrically

$$
\sup \left\{F_{k}^{M}(u, v) ;(u, v) \in[t, 1] \times \mathbf{R}^{+}\right\} \leq c^{2(k+2)} \sup \left\{F_{k}^{N}(u, v) ;(u, v) \in[t, 1] \times \mathbf{R}\right\} .
$$

Thus $c^{-2(k+2)} R_{k}^{M}(t) \leq R_{k}^{N}(t) \leq c^{2(k+2)} R_{k}^{M}(t), t \in(0,1]$.

Lemma 3: For any real $a$ and $b, t \in(0,1]$, the inequality

$$
b^{k+1} M(a) \leq(M(a)+M(a b / t)) R_{k}^{M}(t)
$$

holds.

Proof: If $b / t \leq 1$, then (7) implies

$$
\left(b^{k+1} / R_{k}^{M}(t)-1\right) M(a) \leq\left((b / t)^{k+1}-1\right) M(a) \leq 0 \leq M(a b / t) .
$$


Suppose now $b / t>1$. Obviously $t \leq t / b<1$, and (9) immediately follows from

$$
r_{k}^{M}(t) \geq t \frac{M(a)}{(t / b)^{k+1} M(a b / t)}=\frac{b^{k+1} M(a)}{t^{k} M(a b / t)}
$$

Thus Lemma 3 is proved.

Lemma 4: Let $M \in A C^{k}$. Then for any real $u, v$ with $u^{2}+v^{2} \neq 0$ we have

$$
\left|M^{(k)}(u+v)-M^{(k)}(u)\right| \leq c_{1}|v| M(2 c \xi) / \xi^{k+1},
$$

where $c_{1}=2^{k+1} c, \xi=\max (|u|,|v|)$.

Proof: Suppose first that $|v|<|u| / 2$. In this case $\xi=|u|$, and using (2) we have

$$
\begin{gathered}
\left|M^{(k)}(u+v)-M^{(k)}(u)\right|=\left|\int_{u}^{u+v} M^{(k+1)}(t) d t\right| \\
\leq c \int_{\min (|u|,|u+v|)}^{\max (|u|,|u+v|)} \frac{M(c t)}{|t|^{k+1}} d t \leq c \frac{|v| M(c(|u|+|v|))}{(|u|-|v|)^{k+1}} \leq 2^{k+1} c|v| \frac{M(2 c \xi)}{\xi^{k+1}} .
\end{gathered}
$$

If $|v| \geq|u| / 2$, then $|v| / \xi \geq 1 / 2$ and using (4) and (3) we obtain

$$
\begin{gathered}
\left|M^{(k)}(u+v)-M^{(k)}(u)\right| \leq c\left(\frac{M(c(u+v))}{|u+v|^{k}}+\frac{M(c u)}{|u|^{k}}\right) \\
\leq \frac{c|v|}{2^{k-1} \xi^{k+1}}\left(\left(\frac{2 \xi}{|u+v|}\right)^{k} M(c(u+v))+\left(\frac{2 \xi}{|u|}\right)^{k} M(u)\right) \leq 2^{2-k} c|v| \frac{M(2 c \xi)}{\xi^{k+1}} .
\end{gathered}
$$

Thus Lemma 4 is proved.

We associate to every $M \in A C^{k}$ and $x \in X=L_{M}(S, \Sigma, \mu)$ the symmetric $i$-linear forms $(i=1,2, \ldots, k)$ defined by

$$
\bar{M}_{i}\left(x ; y_{1}, y_{2}, \ldots, y_{i}\right)=\int_{S} M^{(i)}(x(s)) \prod_{k=1}^{i} y_{k}(s) d \mu(s)
$$

and the symmetric $(i-j)$-linear forms $(0 \leq j<i)$

$$
\widetilde{M}_{i, j}\left(x ; y_{1}, y_{2}, \ldots, y_{i-j}\right)=\int_{S} M^{(i)}(x(s)) x^{j}(s) \prod_{k=1}^{i-j} y_{k}(s) d \mu(s),
$$

where $y_{1}, y_{2}, \ldots, y_{i} \in X$. Obviously $\widetilde{M}_{i, 0}=\widetilde{M}_{i}$ and $M_{0}=\widetilde{M}$.

Lemma 5: Let $M \in A C^{k}$. Then $\widetilde{M}_{i, j}(x) \in B^{i-j}(X)$ for every $x \in X$ $(i=1,2, \ldots, k ; 0 \leq j<i)$ and

$$
\left\|\widetilde{M}_{i, j}(x)\right\| \leq c^{k+1}(\widetilde{M}(c x)+c) .
$$


Proof: It is sufficient to show that for fixed $x \in X$

$$
\sup \left\{\left|\widetilde{M}_{i, j}\left(x ; h^{(i-j)}\right)\right| ;\|h\| \leq 1 / c\right\}<\infty(i=1,2, \ldots, k ; 0 \leq j<i) .
$$

Denote $S_{1}=\{s \in S ; 0 \leq|x(s)| \leq|h(s)|\}$ and $S_{2}=S \backslash S_{1}$. Using (3) and (4) we have

$$
\begin{aligned}
\left|\bar{M}_{i, j}\left(x ; h^{(i-j)}\right)\right| & \leq \int_{S}\left|M^{(i)}(x(s))\right||x(s)|^{j}|h(s)|^{i-j} d \mu(s) \\
& \leq c\left(\int_{S_{1}} M(c x(s))(|h(s)| /|x(s)|)^{i-j} d \mu(s)+\int_{S_{2}} M(c x(s)) d \mu(s)\right) \\
& \leq c^{2} \int_{S_{1}} M(c h(s)) d \mu(s)+c \int_{S_{2}} M(c x(s)) d \mu(s) \leq c(\bar{M}(c x)+c) .
\end{aligned}
$$

The next lemma essentially shows that $M^{(k)} \in H^{\omega}\left(\mathbf{R}^{+}\right), \omega(t)=r_{k}^{M}(t)$.

Lemma 6: Let $M \in A C^{k}$. For any real $u, v, w, t$ such that $u^{2}+v^{2} \neq 0,|t| \leq 1 / 4 c$, the incqualities

$$
\left|\left(M^{(k)}(u+t v)-M^{(k)}(u)\right) u^{i} w^{k-i}\right| \leq c_{2}(M(4 c u)+M(v)+M(w)) r_{k}^{M}(|t|)
$$

for $0 \leq i \leq k-1$ and

$$
\left|\left(M^{(k)}(u+t v)-M^{(k)}(u)\right) u^{k}\right| \leq c_{2}(M(4 c u)+M(v)) r_{k}^{M}(|t|)
$$

hold, where $c_{2}=2 c_{1}(2 c)^{k+1}$.

Proof: Lemma 4 implies

$$
\left|\left(M^{(k)}(u+t v)-M^{(k)}(u)\right) u^{i} w^{k-i}\right| \leq c_{1}|t v||w|^{k-i} M(2 c \xi) / \xi^{k+1-i}
$$

where $\xi=\max (|u|,|t v|)$. It is clear that to estimate the right-hand side of (11) it suffices to consider only positive $u, v, w, t$, and $M(1)=1$. We separate the following cases:

a) $w \leq 2 c \xi$. Using (5) and (6) we obtain

$$
\begin{gathered}
t v w^{k-i} M(2 c \xi) / \xi^{k+1-i} \leq(2 c)^{k+1-i} t v M(2 c \xi) /(2 c \xi) \\
\leq(2 c)^{k+1-i} t(M(2 c \xi)+M(v)) \leq 3(2 c)^{k+1-i} t(M(4 c u)+M(v)) .
\end{gathered}
$$

b) $2 c \xi \leq w \leq 2 c \xi / t$. Now

$$
\frac{t v u^{k-i} M(2 c \xi)}{\xi^{k+1-i}} \leq(2 c)^{k+1-i} t\left(\left(\frac{v}{2 c \xi}\right)^{k+1-i}+\left(\frac{w}{2 c \xi}\right)^{k+1-i}\right) M(2 c \xi) .
$$

If $i \neq 0$ using once more (5) and (6) we obtain

$$
\begin{aligned}
\frac{t v w^{k-i} M(2 c \xi)}{\xi^{k+1-i}} & \leq(2 c)^{k+1-i} t(2 M(2 c \xi)+M(v)+M(w)) \\
& \leq 2(2 c)^{k+1-i} t(M(4 c u)+M(v)+M(w)) .
\end{aligned}
$$


If $i=0$ we continue the estimation in (12) using Lemma 3:

$$
\begin{aligned}
\frac{t v w^{k-i} M(2 c \xi)}{\xi^{k+1-i}} & \leq(2 c)^{k+1} t^{-k}\left(\left(\frac{t v}{2 c \xi}\right)^{k+1}+\left(\frac{t w}{2 c \xi}\right)^{k+1}\right) M(2 c \xi) \\
& \leq(2 c)^{k+1}(2 M(2 c \xi)+M(v)+M(w)) r_{k}^{M}(t) \\
& \leq 2(2 c)^{k+1}(M(4 c u)+M(v)+M(w)) r_{k}^{M}(t) .
\end{aligned}
$$

Finally we consider

c) $w \geq 2 c \xi / t$. This case is quite easy. Indeed

$$
\begin{aligned}
\frac{t v w^{k-i} M(2 c \xi)}{\xi^{k+1-i}} & \leq\left(\frac{w}{\xi}\right)^{k-i} M(2 c \xi)=(2 c)^{k-i} t^{i-k}\left(\frac{w t}{2 c \xi}\right)^{k-i} M(2 c \xi) \\
& \leq(2 c)^{k-i} t^{i-k} M(w t) \leq(2 c)^{k-i} t^{i} r_{k}^{M}(t) M(w)
\end{aligned}
$$

where we used (8) in the last inequality.

Combining the estimates obtained in the cases a), b) and c) it is easy to get (10) with $c_{2}=2 c_{1}(2 c)^{k+1}$. The proof of $\left(10^{\prime}\right)$ is the same. Thus Lemma 6 is proved.

Corollary 1: Let $M \in A C^{k}$. Then for every $x, h \in X$ and any $t \in(0,1 / 4 c)$ the estimate

$$
\left|\widetilde{M}(x+t h)-\sum_{j=0}^{k} \frac{t^{j}}{j !} \widetilde{M}_{j}\left(x ; h^{(j)}\right)\right| \leq c_{3}(\widetilde{M}(4 c x)+\widetilde{M}(h)) R_{k}^{M}(t),
$$

holds, where $c_{3}=2 c_{2} / k !$.

Proof: Obviously, for every $s \in S$ :

$$
\left|M(x(s)+\operatorname{th}(s))-\sum_{j=0}^{k} \frac{(t h(s))^{j}}{j !} M^{(j)}(x(s))\right| \leq \frac{|t h(s)|^{k}}{k !}\left|M^{(k)}\left(x(s)+\theta_{s} t h(s)\right)-M^{(k)}(x(s))\right|
$$

for some $\theta_{s} \in(0,1)$. Lemma 6 applied for $i=0, u=x(s), v=\theta_{s} h(s), w=h(s)$ gives

$$
\left|M(x(s)+t h(s))-\sum_{j=0}^{k} \frac{(t h(s))^{j}}{j !} M^{(j)}(x(s))\right| \leq \frac{2 c_{2}}{k !}(M(4 c x(s))+M(h(s))) R_{k}^{M}(t) .
$$

Now to obtain (13) we only have to integrate over $S$ the last inequality.

Remark 1: Corollary 1 is a quantitative improvement of Lemma 4 in [10], where only $o\left(|t|^{k}\right)$ instead of $|t|^{k} r_{k}^{M}(|t|)$ in the right-hand side of (13) was given. The estimate (13) implies, of course, that $\widetilde{M}_{i}: X \rightarrow B^{i}(X)$ is $(k-i)$-times differentiable in $X$ for $i=0, \ldots, k-1$ $\left(B^{0}(X)=\mathbf{R}^{+}\right)$and $D^{j} \bar{M}=\widetilde{M}_{j}(j=1,2, \ldots, k), D^{i} \bar{M}_{j}=\bar{M}_{i+j}(i+j \leq k)$.

Corollary 2: We have $\widetilde{M}_{k, j} \in H^{\omega}\left(X, B^{k-j}(X)\right)(j=0,1, \ldots, k)$, where $\omega(t)=r_{k}^{M}(t)$. 
Proof: We shall prove that for any $y, z$ from the ball $B(x ; 1 / 8 c)$ the inequalities

$$
\begin{gathered}
\left\|\widetilde{M}_{k, j}(y)-\widetilde{M}_{k, j}(z)\right\| \leq c_{4} \varphi(x) r_{k}^{M}(\|y-z\|), j=0,1,2, \ldots, k-1, \\
\left|\widetilde{M}_{k, k}(y)-\widetilde{M}_{k, k}(z)\right| \leq c_{4} \varphi(x) r_{k}^{M}(\|y-z\|),
\end{gathered}
$$

where $c_{4}=\kappa 8^{k-2} c^{2 k} c_{2}, \varphi(x)=(8 c\|x\|+1)^{k-2}(\widetilde{M}(8 c x)+8 c+1)$, hold. Indeed, let $h \in S(X)$, $s \in S$. Obviously

$$
\begin{aligned}
\mid \bar{M}_{k, j}\left(y ; h^{(k-j)}\right) & -\widetilde{M}_{k, j}\left(z ; h^{(k-j)}\right) \mid \\
& \leq\left|\int_{S} M^{(k)}(y(s))\left(y^{j}(s)-z^{j}(s)\right) h^{k-j}(s) d \mu(s)\right| \\
& +\int_{S}\left|M^{(k)}(y(s))-M^{(k)}(z(s))\right||z(s)|^{j}|h(s)|^{k-j} d \mu(s) .
\end{aligned}
$$

The second member of the last sum is easily estimated for $j<k$ using (10) for $u=z(s)$, $v=((y(s)-z(s))) /\|y-z\|, t=\|y-z\|, w=h(s)$ and $(6):$

$$
\begin{aligned}
& \left.\int_{S}\left|M^{(k)}(y(s))-M^{(k)}(z(s))\right||z(s)|^{j} \cdot h(s)\right|^{k-j} d \mu(s) \\
& \leq c_{2}(\widetilde{M}(4 c z)+\widetilde{M}((y-z) /\|y-z\|)+\bar{M}(h)) r_{k}^{M}(\|y-z\|) \\
& \leq \frac{c_{2}}{2}(\widetilde{M}(8 c x)+5) r_{k}^{M}(\|y-z\|) .
\end{aligned}
$$

To obtain (14) for $j<k$ with $c_{4}=\kappa 8^{k-2} c^{2 k} c_{2}$ and $\varphi(k)=(8 c\|x\|+1)^{k-1}(\widetilde{M}(8 c x)+8 c+1)$, it is enough to estimate the first member of (15) in the following way:

$$
\begin{aligned}
& \left|\int_{S} M^{(k)}(y(s))\left(y^{j}(s)-z^{j}(s)\right) h^{k-j}(s) d \mu(s)\right| \\
& \leq\left\|\widetilde{M}_{k}(y)\right\|_{1}\|y-z\| \sum_{i=0}^{j-1}\|y\|^{i}\|z\|^{j-i-1}\|h\|^{k-j} \\
& \leq \kappa c^{k+1}(\widetilde{M}(c y)+c)(\|x\|+1 / 8 c)^{j-1}\|y-z\| \\
& \leq \kappa 8^{k-1} c^{2 k}\left(\frac{\widetilde{M}(2 c x)+\widetilde{M}(2 c(y-x))}{2}+c\right)(8 c\|x\|+1)^{k-2}\|y-z\| \\
& \leq \kappa 8^{k-2} c^{2 k}(4 \widetilde{M}(2 c x)+8 c+1)(8 c\|x\|+1)^{k-2}\|y-z\| .
\end{aligned}
$$

We used Lemma 5 , the relation between the norms $\|\cdot\|$ and $\|\cdot\|_{1}$ in $B^{k}(X)$ and the convexity of $M$. The proof of $\left(14^{\prime}\right)$ uses $\left(10^{\prime}\right)$ and is practically the same.

Remark 2: Obviously $\widetilde{M}_{j} \in H^{1}\left(X, B^{j}(X)\right)$ and $\widetilde{M}_{i, j} \in H^{1}\left(X, B^{i-j}(X)\right)$ for $0 \leq j \leq i<k$. 


\section{Main result}

We are ready to prove the following

Theorem 1: Let $M \in A C^{k}$ and $(S, \Sigma, \mu)$ be a measure space. Thén $X=H_{M}(S, \Sigma, \mu)$ is $H^{\omega}$-smooth, where $\omega(t)=R_{k}^{M}(t)$.

Proof: Set $n(x)=\|x\|$. Using Remark 1 and the implicit function theorem applied to 'the equation $\widetilde{M}(x / n(x))-1=0$ we obtain as in [10, Theorem 6] $n^{\prime}(x)$ $=\widetilde{M}_{1,0}(x / n(x)) / \widetilde{M}_{1,1}(x / n(x))$, which, by an easy induction argument, implies that $n$ is $k$-times differentiable in $X \backslash\{0\}$. What we have to prove in addition is that $n^{(k)} \in H^{\omega_{1}}\left(X \backslash 0, B^{k}(X)\right)$, $\omega_{1}=r_{k}^{M}$. To this end we need some more information about the $k$-th derivative of the norm.

First for sake of brevity we introduce the notation

$$
\bar{M}_{i, j}(x)=\widetilde{M}_{i, j}(x / n(x)) .
$$

Using the equality

$$
D\left(\frac{x}{n(x)}\right)(y)=\frac{y}{n(x)}-\frac{x}{n^{2}(x)} D n(x ; y)=\frac{y \bar{M}_{1,1}(x)-\bar{M}_{1,0}(x ; y)}{n(x) \bar{M}_{1,1}(x)},
$$

we obtain by induction

$$
n^{(k)}(x)=\frac{\sum_{i=0}^{k} C_{k}^{i}(-1)^{i} \bar{M}_{k, i}(x) \bar{M}_{1,1}^{k-i}(x) \bar{M}_{1,0}^{i}(x)+P\left(\bar{M}_{i, j}(x)\right)}{n^{k-1}(x) \bar{M}_{1,1}^{k+1}(x)},
$$

where $P\left(\bar{M}_{i, j}(x)\right)$ is a polynomial with respect to $\bar{M}_{i, j}(i<k)$ and $P\left(\bar{M}_{i, j}(x)\right) \in B^{k}(X)$ for fixed $x$.

Let $\omega_{1}=r_{k}^{M}$. It is easy to check that $f \in H^{\omega_{1}}\left(X, B^{k}(X)\right), g \in H^{1}\left(x, \mathbf{R}^{+}\right)$imply $f / g \in$ $H^{\omega_{1}}\left(X \backslash A ; B^{k}(X)\right)$, where $A=\{x \in X: g(x)=0\}$. Indeed, fix $x \notin A$. Then for sufficiently small $\delta>0$,

$$
\left\|\frac{f(y)}{g(y)}-\frac{f(z)}{g(z)}\right\| \leq 6 \frac{\|f(y)-f(z)\|\|g(x)\|+\|g(y)-g(z)\|\|f(x)\|}{\|g(x)\|^{2}},
$$

for any $y, z \in B(x ; \delta)$. Let now $x \neq 0, r=\min (\|x\| / 2,1 / 8 c)$. As

$$
\left\|\frac{y}{\|y\|}-\frac{\dot{z}}{\|z\|}\right\| \leq 4 \frac{\|y-z\|}{\|x\|}
$$

for $y, z \in B(x ; r)$, from (14) and $\left(14^{\prime}\right)$ and Lemma 1 it follows for any $y, z \in B(x ; r)$ that

$$
\begin{aligned}
& \left\|\bar{M}_{k, j}(y)-\bar{M}_{k, j}(z)\right\| \leq c_{4} \varphi(x) r_{k}^{M}\left(\left\|\frac{y}{\|y\|}-\frac{z}{\|z\|}\right\|\right) \\
& \leq c_{4} \varphi(x) \max \left(\frac{4}{\|x\|}, 1\right) r_{k}^{M}(\|y-z\|)(j=0,1, \ldots, k) .
\end{aligned}
$$

Obviously $n$ and $\bar{M}_{i, j}$ are in $H^{1}\left(X, B^{i-j}(X)\right)$ for every $i=1,2 \ldots, k-1,0 \leq j \leq i$ and therefore $n \bar{M}_{1,1} \in H^{1}\left(X, \mathbf{R}^{+}\right)$. Now from the representation (15) of $n^{(k)},(16)$ and (17) it follows that $n^{(k)} \in H^{\omega_{1}}\left(X \backslash\{0\}, B^{k}(X)\right)$. Thus Theorem 1 is proved. 


\section{Smooth renormings in arbitrary Orlicz spaces}

We shall treat in details only the case of sequence spaces. The cases $H_{M}(0,1), H_{M}(0, \infty)$, and the general case $H_{M}(S, \Sigma, \mu)$ can be analogously treated. It is not hard to see that, without some kind of smoothness of the Orlicz function $M$, the condition $\alpha_{M}^{0}>1$ cannot ensure the differentiability of the usual norm in $h_{M}$. Nevertheless, equivalent smooth Orlicz renorming, i.e. one generated by a suitable Orlicz function, equivalent to $M$ at 0 , is possible. Namely, the following theorem holds true.

Theorem 2: Let $\alpha_{M}^{0} \in(1, \infty)$. Then in $h_{M}$ there exists an equivalent $H^{\omega}{ }^{\omega}$-smooth norm, where $\omega(t)=R_{k,[0,1]}^{M}(t), k=E\left(\alpha_{M}^{0}\right)$.

Proof: As usual we suppose $M(1)=1$, and since the behaviour of $M$ at $\infty$ is unessential, we "correct" it near $\infty$ to a function $N$ in such a way that $\alpha_{N}=\alpha_{M}^{0}$. For example:

$$
N(t)=\left\{\begin{array}{ll}
M(t), & t \in[0,1] \\
t^{k+1}, & t \in[1, \infty)
\end{array} .\right.
$$

Put $N_{2}(t)=\int_{0}^{t} N_{1}(u) \exp (u /(u-t)) d u / u$, where $N_{1}(t)=\int_{0}^{t} N(u) d u / u$. It is not hard to check that

$$
(1 / 4) N(t / 4) \leq e^{-1} N(t / 4) \leq N_{2}(t) \leq N(t), t \in[0, \infty) .
$$

This implies that $h_{N_{2}}$ is isomorphic to $h_{M}$. Moreover, it is easily verified that $N_{2} \in A C^{k}$ for any $k$, and we may apply Theorem 1: $h_{N_{2}}$ is $H^{\omega}$-smooth, $\omega(t)=R_{k}^{N_{2}}(t), k=E\left(\alpha_{N_{2}}\right)=E\left(\alpha_{M}^{0}\right)$.

Lemma 2 and (19) imply

$$
4^{-2(k+2)} R_{k}^{N}(t) \leq R_{k}^{N_{2}}(t) \leq 4^{2(k+2)} R_{k}^{N}(t), t \in[0,1] .
$$

To finish we observe that $R_{k}^{N}=R_{k,[0,1]}^{M}$. Indeed, $R_{k}^{N} \geq R_{k,[0,1]}^{M}$ is obvious, and we only have to prove $R_{k}^{N} \leq R_{k,\{0,1]}^{M}$. Analogously to the proof of Lemma 2 we show that for any $(u, v) \in[t, 1] \times \mathbf{R}^{+}$ there are $u_{1}$ and $v_{1},\left(u_{1}, v_{1}\right) \in[t, 1] \times[0,1]$ with $F_{k}^{N}(u, v)=F_{k}^{M}\left(u_{1}, v_{1}\right)$. If $v \leq 1$ we take simply $u_{1}=u, v_{1}=v$. Let $v>1$ and $u v \leq 1$. Then

$$
F_{k}^{N}(u, v)=(u v)^{-(k+1)} M(u v)=F_{k}^{M}\left(u_{1}, v_{1}\right), u_{1}=u v, v_{1}=1
$$

Finally, if $u v>1$, then $F_{k}^{N}(u, v)=1=F_{k}^{M}\left(u_{1}, v_{1}\right), u_{1}=v_{1}=1$. Theorem 2 is proved $\bullet$

Remark 3: This result is of no interest if $M \sim t^{2 p}$ at $0, p \in \mathrm{N}$, because it is well known that in $l_{2 p}$ the usual norm is infinitely many times differentiable. On the other hand if $M \not t^{2 p}$ at $0, p \in N$, the best order of smoothness in $h_{M}$ by equivalent renorming is not better than $t^{\alpha_{M}^{0}}$. Indeed, for $\alpha_{M}^{0} \neq 2 p, p \in \mathrm{N}$ this follows simply from the fact that $l_{\alpha_{M}^{0}}$ is isomorphic to a subspace of $h_{M}$, if we combine this with the result from [1] formulated in the Introduction. If $\alpha_{M}^{0}=2 p, p \in \mathrm{N}$, but $M \nsim t^{2 p}$ at 0 , it was shown in [11] that in $h_{M}$ there is no equivalent $\alpha_{M}^{0}$-times differentiable norm, i.e. any equivalent norm in $h_{M}$ is again at most $H^{\alpha_{M}^{0} \text {-smooth. }}$

Corollary 3: Let $M \not t^{2 p}$ at $0, p \in N$. Then 
a) the order of smoothness $R_{k,[0,1]}^{M}, k=E\left(\alpha_{M}^{0}\right)$ cannot be improved with respect to power type orders;

b) if

$$
M(u v) \leq c u_{M}^{\alpha_{M}^{0}} M(v), u, v \in[0,1]
$$

then in $h_{M}$ there is an $H^{\alpha_{M}^{0}}$-smooth norm, i.e.norm of best order of smoothness.

Proof: To obtain a) it is sufficient to observe that from the definition of $\alpha_{M}^{0}$ it follows for any $u, v \in[0,1]$ and fixed $\varepsilon>0$ that $M(u, v) \leq c_{\varepsilon} u^{\alpha_{M}^{0}-\varepsilon} M(v)$, for some $c_{\varepsilon}>0$, which implies for $k=E\left(\alpha_{M}^{0}\right)$ that $R_{k,[0,1]}^{M}(t) \leq c_{\varepsilon} t^{\alpha_{M}^{0}-\varepsilon}, t \in[0,1]$.

b) In this case $R_{k,[0,1]}^{M}(t) \leq c t^{\alpha_{M}^{0}}, k=E\left(\alpha_{M}^{0}\right)$

Remark 4: The condition (20) is fulfilled for example if $M\left(t^{1 / \alpha_{M}^{0}}\right)$ is quasi-convex. Results analogous to those from Theorem 2 and Corollary $3 \mathrm{~b}$ ) can be obtained for the function spaces $H_{M}(0,1)$ and $H_{M}(0, \infty)$ and for general Orlicz spaces $H_{M}(S, \Sigma, \mu)$, as well, using the same techniques and results on embeddings of $l_{p}$ spaces in Orlicz function spaces [8]. The corresponding orders of smoothness for $H_{M}(0,1)$ and $H_{M}(0, \infty)$ are respectively

$$
\Re_{k}^{M}(t)=t^{k+1} \sup \left\{1 / F_{k}^{M}(u, v) ; u \in[1,1 / t], v \in[1, \infty)\right\}, k=E\left(\alpha_{M}^{\infty}\right)
$$

and $R_{k}^{M}(t), k=E\left(\alpha_{M}\right)$.

Remark 5: Very probably the orders of smoothness from Theorem 2 and Remark 4 are the best ones in general as they agree with those from [10] for the cases $\alpha_{M}^{0}, \alpha_{M}^{\infty}, \alpha_{M} \in(1,2)$ that are the best possible up to arbitrary (not only Orlicz) equivalent renorming (see $[3,4]$ ).

Finally we give some examples.

Examples: Let $M(t)=t^{p}(1+|\ln t|)^{q}, p>1$. Obviously $M$ satisfies the $\Delta_{2}$-condition at 0 and at $\infty$ and $\alpha_{M}^{0}=\alpha_{M}^{\infty}=p$. Therefore $h_{M}=l_{M}, H_{M}(0,1)=L_{M}(0,1)$ and

a) if $q<0$ :

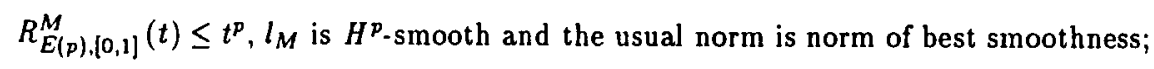

$\Re_{E(p)}^{M}(t) \leq 2 / M(1 / t)$ for sinall $t$ and $L_{M}(0,1)$ is $H^{M}$-smooth.

b) if $q>0$ :

$R_{E(p),\{0,1]}^{M}(t) \leq 2 M(t)$ for small $t$ and $l_{M}$ is $H^{M}$-smooth;

$\Re_{E(p)}^{M} \leq c t^{p}$ and $L_{M}(0,1)$ is $H^{p}$-smooth and the usual norm is norm of best smoothness. 


\section{REFERENCES}

[1] BONIC, R. and J. FRAMPTON: Smooth functions on Banach manifolds. J. Math. Mech. 15 (1966), 877 - 898.

[2] DEVILLE, R.: A characterization of $H^{\infty}$-smooth Banach spaces. Bull. London Math. Soc. $22(1990), 13-17$.

[3] FIGIEL, T.: On the moduli of convexity and smoothuoss. Studia Math. 56 (1976), $121-155$.

[4] FIGIEL, T. and G. PISIER: Series aleatoires dans les espace uniformement convexes ou uniformement lisses. C. R. Acad. Sci. Paris (Serie A) 279 (1974), 611 - 614.

[5] KRASNOSELSKII, M. and YA. RUTICKII : Convex Functions and Orlicz Spaces. Groningon: Wolters-Noordhoff 1961.

[6] LiNDENSTRAUSS, J. and L.TZAFRIRI: Classical Banach Spaces. Vol. II: Sequence Spaees. Berlin - Heidelberg - Now York: Springer - Verlag 1979.

[7] LINDENSTRAUSS, J. and L. TZAFRIRI: On Orticz sequence spaces I. Isr. J. Math. 10 (1971), $379-390$.

[8] LINDENSTRAUSS, J. and L.TZAFRIRI: On Orlicz sequence spaces III. Isr. J. Math. 14 (1973), 368 - 389.

[9] MALEEV, R.P. and S. L. TROYANSKI: On the moduli of convexity and smoothness in Orlicz spaces. Studia Math. 54 (1975), 131 - 141.

[10] MALEEV, R. P. and S. L. TROYANSKI: Smooth norms in Orlicz spaces. Can. Bull. Math. 34 (1991), 74 - 82.

[11] MALEEV, R. P. and S. L. TROYANSKI: Smooth functions in Orlicz spaces. In: Banach Space Theory. Proc. Res. Workshop, July 5 - 25, 1987. Contemp. Math. 85 (1989), 355 - 370.

[12] SUNDARESAN, K.: Smooth Banach spaces. Math. Ann. 173 (1967), 191 - 199.

[13] SUNDARESAN, K. and S. SWAMINATHAN: Geometry and Analysis in Banach Spaces. Lect. Notes Math: 1131 (1985), 1 - 